\title{
Flow-induced delayed Freedericksz transition
}

\author{
N. J. Mottram and G. McKay \\ Department of Mathematics and Statistics, University of Strathclyde, Glasgow G1 1XH, United Kingdom \\ C. V. Brown, C. T. Russell, I. C. Sage, and C. Tsakonas \\ School of Science and Technology, Nottingham Trent University, Nottingham NG11 8NS, United Kingdom
}

(Received 25 September 2015; published 15 March 2016)

\begin{abstract}
We demonstrate that a compact manometer experiment allows direct observation of a delay to the classical electric-field-induced Freedericksz transition produced by flow in a highly dispersive nematic liquid crystal layer. The Ericksen-Leslie equations are used to show that a flow aligning torque generated in the nematic layer under Poiseuille flow competes with the orthogonal electric-field reorientation torque. This model fully reproduces the experimental results using only self-consistently determined viscosity values, and predicts a more generally applicable expression for the dependence of the delay $E_{c} \propto \sqrt{\zeta / \Delta \chi_{e}}$ on the shear rate $\zeta$ and on the electric susceptibility anisotropy $\Delta \chi_{e}$.
\end{abstract}

DOI: 10.1103/PhysRevE.93.030701

Within anisotropic liquids, alignment of the anisotropic axis may occur in a number of ways, for instance, through surface interactions, viscous stress, and external applied electric or magnetic fields. Nematic liquid crystals are important examples of such materials, where the orientation of the average molecular direction, the director, is affected by, and affects, both the flow properties and electric-field effects [1,2]. Understanding the complex interplay between molecular alignment and flow properties is particularly important in liquid crystal displays, where director-flow feedback can be detrimental [3], as well as for recently proposed photonic device and electronic paper liquid crystal technologies based on droplet manipulation via electrowetting or dielectrophoresis [4,5]. The director provides a symmetry-breaking axis which leads to an asymmetric fluid stress tensor, an anisotropic electric polarization tensor, as well as internal elasticity [6-10]. There are three important features of these effects that we investigate here: first, the director-shear stress interaction tends to orient the director towards a preferred "flow alignment" angle relative to the flow velocity direction [9]; secondly, an applied electric field also tends to reorient the director, an effect known as the Freedericksz transition, and may compete with the flow alignment effect; thirdly, due to the director-electric-field interaction and the asymmetric fluid stress tensor, flowing nematic liquid crystals tend to exhibit an effective viscosity that depends on the magnitude of an applied electric field [11,12]. Such electrorheological effects have been previously observed in positive dielectric anisotropy materials during forced flow through channels or shear flow between concentric cylindrical plates in a viscometer instrument [13-17].

In this Rapid Communication we consider, experimentally and theoretically, gravitationally driven flow of a nematic liquid crystal layer in a rectangular cross-section manometer, shown in Fig. 1, which provides a continuous range of different

Published by the American Physical Society under the terms of the Creative Commons Attribution 3.0 License. Further distribution of this work must maintain attribution to the author(s) and the published article's title, journal citation, and DOI. flow rates within a single experimental run. An ac voltage is applied across the thinnest dimension of the liquid slug during flow towards the equilibrium state, which is achieved when the heights of the liquid columns in the two arms of the manometer become equal. Using a highly dispersive "dual frequency" commercial nematic liquid crystal material MLC2048 (Merck $\mathrm{KGa}$ ) additionally allows the calculation of two limiting Miesowicz viscosity values. One corresponds to an electric field which induces alignment of the anisotropic axis parallel to the field direction and therefore perpendicular to the flow direction, when an ac voltage at $1 \mathrm{kHz}$ is applied (when the dielectric anisotropy $\Delta \epsilon>0$, or equivalently the electric susceptibility anisotropy $\left.\Delta \chi_{e}>0\right)$. The other viscosity is calculated when the field-induced alignment is perpendicular to the field and therefore parallel to the flow direction, with an ac voltage of $50 \mathrm{kHz}$ applied (when $\Delta \epsilon<0$ ) [18]. At these frequencies the director responds to the rms voltage, which we denote by $V$.

The nematic liquid crystal slug of length $L=0.072 \mathrm{~m}$ is sandwiched in the $y$ direction in a gap of size $w=$ $170 \times 10^{-6} \mathrm{~m}$ between two glass plates which are coated with transparent conducting oxide electrodes and a thin $\left(<10^{-6} \mathrm{~m}\right)$ dielectric layer of SU-8 photoresist (MicroChem Corp., Newton, MA, USA). SU-8 was also used to fabricate the sides of the manometer channel in the $x-z$ plane, where $d=$ $3.5 \times 10^{-3} \mathrm{~m}$. At equilibrium, depicted in Fig. 1(a), planar degenerate anchoring at the SU-8 surfaces aligns the director parallel to the long axis of the U-tube, the direction of the flow velocity. A suction-generated pressure difference between the two arms results in an initial height difference $h(0)$ between the two menisci, shown in Fig. 1(b). The ac voltage is then applied, the pressure difference is removed, and flow under gravity reduces the height difference $h(t)$ with time towards the equilibrium value, $h=0$, as $t \rightarrow \infty$. At early times when the height difference is large, the flow speeds and, therefore, shear stresses will be large; while at late times, when the height difference is small, the system tends towards equilibrium, and flow speeds and shear stresses decay to zero. In this way we may investigate the influence of a range of shear stresses on the director orientation within a single experimental run.

Figure 2 shows the experimental values of $\ln [h(t) / h(0)]$ for the cases (a) $\Delta \epsilon<0$ and (b) $\Delta \epsilon>0$, and for rms voltages 


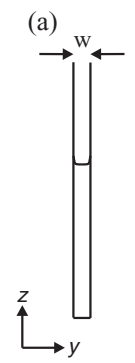

Side view

(b)

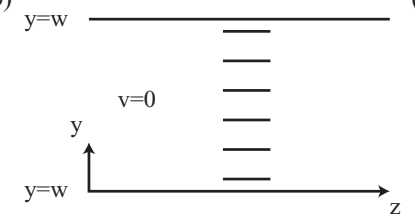

no flow and no electric field

$\longrightarrow$ no director distortion (c)

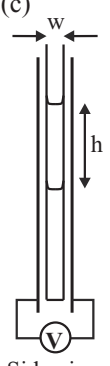

Side view

(d)

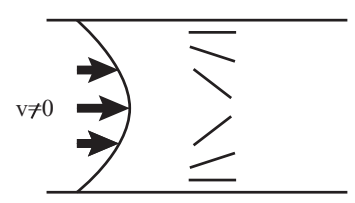

flow and electric field applied $\longrightarrow$ director distortion
FIG. 1. Experimental setup: $(a, b)$ with no applied field and no difference in height between the arms of the manometer, there is no flow and no director distortion; (c, d) with an applied electric field and a difference in heights, there will be director distortion due to both the flow and electric-field effects.

from 1 to $20 \mathrm{~V}$, which for a Newtonian liquid would be a single straight line. However, for both positive and negative dielectric anisotropies, it is clear that the evolution of $h(t)$, and therefore the flow rate and effective viscosity, is voltage and time dependent. This behavior may be explained as follows:

(1) at a high voltage in a material where $\Delta \epsilon<0$ [A in Fig. 2(a)], the director aligns perpendicular to the field, and therefore parallel to the substrate, and in the direction of the flow velocity throughout the device;

(2) at low voltages and $\Delta \epsilon<0$ [B in Fig. 2(a)] flow dominates and causes director flow alignment but, for a small flow alignment angle, the director remains close to parallel to the substrate and $h(t)$ does not change considerably from the high voltage case;

(3) at a high voltage in a material where $\Delta \epsilon>0$ [C in Fig. 2(b)], the director aligns parallel to the field, and thus perpendicular to the flow velocity, in a large portion of the device;

(4) for lower values of the voltage [D in Fig. 2(b)], flow effects dominate, at least initially when flow shear stress is high, and since a flow-aligned director is almost perpendicular to the field direction, the behavior of $h(t)$ is very different to the high voltage case. However, for late times in this final case, the flow speed, and thus shear stress, decays so that field effects dominate, resulting in behavior [shown by the gradient of the curves in Fig. 2(b)] which is similar to the high voltage situation. We thus have a time-dependent effective viscosity with flow dominance being replaced by field dominance as time progresses.

For our theoretical model, since $d \gg w$, we will assume a one-dimensional system considering distortions to the nematic liquid crystal layer between $y=0$ and $y=w$. The dependent variables are the director angle $\theta(y, t)$ from the $z$ direction, the flow velocity $v(y, t)$ parallel to the plates in the $z$
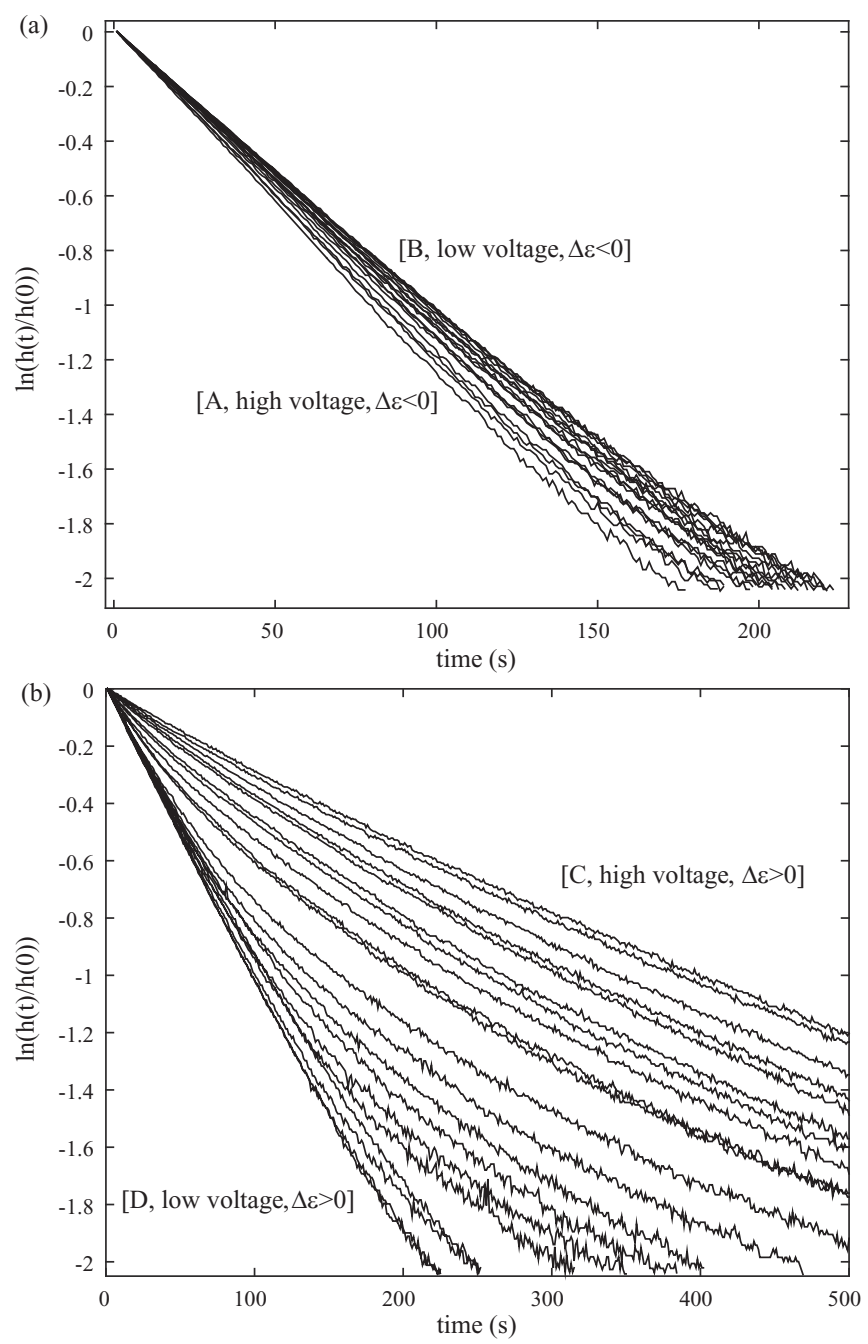

FIG. 2. Experimental results: $\ln [h(t) / h(0)]$ versus time $t$ for (a) negative dielectric anisotropy, $\Delta \epsilon<0$; (b) positive dielectric anisotropy, $\Delta \epsilon>0$.

direction, the pressure in the fluid $p(z, t)$, the electric potential $U(y, t)$, and the height difference between the menisci $h(t)$. The dynamics of the director angle and flow speed can then be modeled as being governed by the Ericksen-Leslie equations [9,19] with the electric potential $U$ determined through the coupling of Maxwell's equations [20] and the height difference determined by a flux condition. We tackle this rather complicated model analytically by integrating the Ericksen-Leslie linear momentum equation along the length of the liquid plug and by considering a situation where the nematic director is uniform throughout the U-tube, aligned through a competition between the electric field and flow, and where the velocity inertial time scale is much shorter than the evolution of the height. This approximation, that the director angle is constant across the full width of the U-tube, is equivalent to neglecting the boundary layers in $\theta(y, t)$ that exist close to the U-tube bounding walls.

If we therefore approximate the director angle by $\theta \equiv \theta_{\mathrm{e}}$, then the electric potential, flow speed, and height evolution are 
found to be

$$
\begin{gathered}
U=\frac{V y}{w}, \\
v=\frac{(\rho \hat{g} h-\Delta p)}{2 L g\left(\theta_{\mathrm{e}}\right)} y(w-y), \\
h=\frac{\Delta p}{\rho \hat{g}}+\left(h(0)-\frac{\Delta p}{\rho \hat{g}}\right) \exp \left(\frac{\rho \hat{g} w^{2}}{12 L g\left(\theta_{\mathrm{e}}\right)} t\right),
\end{gathered}
$$

where

$$
g\left(\theta_{\mathrm{e}}\right)=\eta_{1} \cos ^{2} \theta_{\mathrm{e}}+\eta_{2} \sin ^{2} \theta_{\mathrm{e}}+\eta_{12} \sin ^{2} \theta_{\mathrm{e}} \cos ^{2} \theta_{\mathrm{e}},
$$

and $\eta_{1}, \eta_{2}, \eta_{12}$ are Miesowicz viscosities [21], $\rho$ is fluid density, $\Delta p$ is the difference in pressure at the two menisci, and $\hat{g}=$ $-9.8 \mathrm{~m} \mathrm{~s}^{-2}$ is the acceleration due to gravity.

During the experiment, after the external forcing created by the pressure difference is removed so that $\Delta p=0$, the height difference decays exponentially with

$$
\ln [h(t) / h(0)]=\frac{\rho \hat{g} w^{2}}{12 \operatorname{Lg}\left(\theta_{\mathrm{e}}\right)} t .
$$

Therefore, if the director angle is approximately constant through the sample, the effective viscosity of the liquid crystal $\eta_{\mathrm{e}}=g\left(\theta_{\mathrm{e}}\right)$ can be determined from the gradient of the experimental plot of $\ln [h(t) / h(0)]$ against time, $t$.

In order to use Eq. (5) and the data in Fig. 2 to derive the effective viscosity as a function of voltage and shear rate, we require the relationship between the director angle $\theta_{\mathrm{e}}$ and the voltage and shear rate. For this quasistatic model, we can determine this relationship by making the assumption that the elastic forces in the bulk of the cell are negligible compared to the flow alignment forces and field-induced alignment. However, this assumption is, in fact, equivalent to assuming the director angle is constant through the full width of the U-tube, as we have previously used in deriving Eqs. (1)-(3). With $\zeta$ representing an average shear rate within the cell, the angular momentum balance equation from Ericksen-Leslie theory then simplifies to

$$
0=\frac{\epsilon_{0} \Delta \epsilon V^{2}}{w^{2}} \sin 2 \theta_{\mathrm{e}}-\left[\left(\eta_{1}-\eta_{2}\right) \cos \left(2 \theta_{\mathrm{e}}\right)+\gamma_{1}\right] \zeta,
$$

where $\gamma_{1}$ is the rotational nematic viscosity and $\epsilon_{0}$ is the permittivity of free space. The average shear rate $\zeta$ can be approximated using the quadratic velocity profile (2),

$$
\zeta=\frac{2}{w} \int_{0}^{w / 2} \frac{\rho \hat{g} h}{2 L g\left(\theta_{\mathrm{e}}\right)}(w-2 y) d y=\frac{\rho \hat{g} w}{4 L \eta_{\mathrm{av}}} h,
$$

where $\eta_{\mathrm{av}}$ is an average viscosity in Poiseuille flow. Therefore, given an estimate of the average viscosity, we can estimate the shear rate $\zeta$ at any particular time using the height difference $h(t)$ in the U-tube. For this simple model we use the rather crude, but effective, approximation of $\eta_{\mathrm{av}}=\left(\eta_{1}+\eta_{2}\right) / 2$, the average of the two extreme situations $\theta_{\mathrm{e}}=0$ and $\theta_{\mathrm{e}}=\pi / 2$. Given a value of the shear rate, Eq. (6) provides the bulk equilibrium director angle,

$$
\theta_{\mathrm{e}}=\tan ^{-1}\left[-\delta \pm \sqrt{\left(\delta^{2}+\frac{\left(\eta_{1}-\eta_{2}+\gamma_{1}\right)}{\left(\eta_{1}-\eta_{2}-\gamma_{1}\right)}\right)}\right]
$$

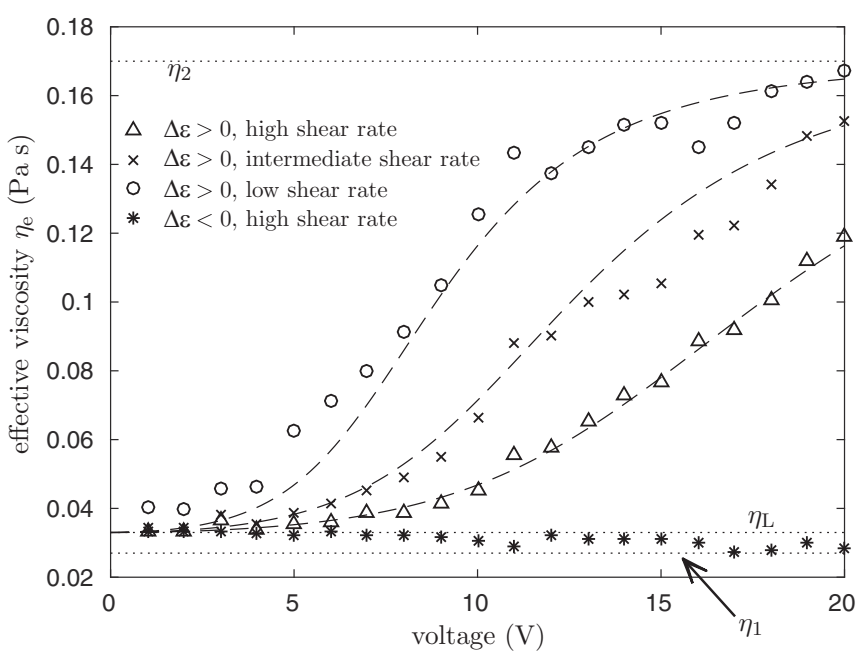

FIG. 3. Experimental (symbols) and theoretical (dashed lines) effective viscosities. The dotted lines indicate viscosities $\eta_{1}, \eta_{2}$, and $\eta_{\mathrm{L}}$ adopted in the theoretical modeling.

where $\delta=\left(\epsilon_{0} \Delta \epsilon V^{2}\right) /\left[w^{2}\left(\eta_{1}-\eta_{2}-\gamma_{1}\right) \zeta\right]$. For large $V$ we see that either $\theta_{\mathrm{e}} \rightarrow \pi / 2$ or $\theta_{\mathrm{e}} \rightarrow 0$. The former is the stable solution for $\Delta \epsilon>0$, while the latter is stable for the $\Delta \epsilon<0$ case. For small $V$ we obtain $\theta_{\mathrm{e}} \approx \theta_{\mathrm{L}}$, the Leslie flow aligning angle defined earlier.

Using Eq. (5) and the data from Fig. 2 for all voltages, we can estimate the experimental effective viscosities using the Curve Fitting Toolbox in MATLAB [22]. From these values of $\eta_{\mathrm{e}}$ we are then able to estimate Miesowicz viscosities: $\eta_{1}=g(0)=0.027 \mathrm{~Pa}$ s is derived from the effective viscosity for the high-frequency ac $(\Delta \epsilon<0)$ plots at high voltages since, for this situation, $\theta_{\mathrm{e}} \approx 0 ; \eta_{2}=g(\pi / 2)=0.170 \mathrm{Pas}$ is derived from the effective viscosity for the low-frequency ac $(\Delta \epsilon>0)$ plots at high voltages since $\theta_{\mathrm{e}} \approx \pi / 2$. Since our analysis neglects the presence of any boundary layer, then this estimate for the viscosity $\eta_{2}$ will be conservative. The viscosity $\eta_{\mathrm{L}}=g\left(\theta_{\mathrm{L}}\right)=0.033 \mathrm{~Pa} \mathrm{~s}=g\left(\theta_{\mathrm{L}}\right)$, where $\theta_{\mathrm{L}}=\tan ^{-1} \sqrt{\left(\eta_{1}-\eta_{2}+\gamma_{1}\right) /\left(\eta_{1}-\eta_{2}-\gamma_{1}\right)}$ is the Leslie flow alignment [21], is derived from the flow dominated region at early times and low voltage. We see that the flow alignment angle is small and $\eta_{L} \sim \eta_{1}$. These estimates for the Miesowicz viscosities are represented by dotted lines in Fig. 3. In this figure we also provide the effective viscosities, plotted as symbols, at four specific instantaneous height differences in the experiments: for $\Delta \epsilon>0$ and $h=0.030 \mathrm{~m}$ (early time), $h=0.015 \mathrm{~m}$ (intermediate time), $h=0.008 \mathrm{~m}$ (late time), and for $\Delta \epsilon<0$ and $h=0.030 \mathrm{~m}$ (early time).

However, Eqs. (4), (5), and (8) together also provide a theoretical relationship between effective viscosity and any height difference and voltage. Given the nematic liquid crystal material parameters [18] for density and dielectric anisotropy, $\rho=1050 \mathrm{~kg} \mathrm{~m}^{-2}, \Delta \epsilon= \pm 3.0 \mathrm{~F} \mathrm{~m}^{-1}$, we have one remaining unknown parameter, the rotational viscosity $\gamma_{1}$ (note: the specification of $\eta_{1}, \eta_{2}, \eta_{L}, \gamma_{1}$ allows the value of $\eta_{12}$ to be determined). The value of $\gamma_{1}=0.1 \mathrm{~Pa}$ s was therefore obtained via a simultaneous fit of the experimental effective viscosity data and the theoretical effective viscosity, using the height differences mentioned above, which correspond to shear rates 
of $\zeta=1.842 \mathrm{~s}^{-1}, 0.921 \mathrm{~s}^{-1}$, and $0.461 \mathrm{~s}^{-1}$. The theoretical relationship between effective viscosity, height difference and shear rate, and voltage is plotted in Fig. 3 (dashed lines), showing remarkably good agreement to the experimental values given only a single fitting parameter has been used. It should be noted that viscosity values for the material MLC2048 have previously been inferred from time-resolved optical measurements of the dynamic switching of the material in a hybrid aligned cell geometry [23]. However, these reported viscosity values are not consistent with our observation that this material exhibits flow alignment behavior of the director at high flow rates.

From Fig. 3 we clearly see that the effect of flow is to delay the Freedericksz transition. The classical critical voltage can be determined from the nematic liquid crystal material parameters given earlier, $V_{c}=\pi \sqrt{K_{1} / \epsilon_{0} \Delta \epsilon} \approx 2.43 \mathrm{~V}$. In the long-time limit, once the flow has decayed and thus the shear stress is low, the effective viscosity is largely determined by the director angle reorientation due to the Freedericksz transition, i.e., the field dominates. However, at intermediate and high shear rates, we see a delay in the Freedericksz transition, with significant reorientation of the director occurring at much higher voltages. We can define a measure of the delay as being the electric-field strength at which the inflection point in the effective viscosity occurs in Fig. 3. We find this to be $E_{c}=$ $\Gamma\left(\eta_{1}, \eta_{2}, \eta_{12}, \gamma_{1}\right) \sqrt{\zeta /\left(\epsilon_{0} \Delta \chi_{\mathrm{e}}\right)}$, where $\Delta \chi_{\mathrm{e}}$ is the susceptibility anisotropy and $\Gamma$ is a function of the viscosities that may be calculated analytically by determining $V_{c}\left(=-w E_{c}\right)$ from $d^{2} \eta_{\mathrm{e}} / d V^{2}=0$, using Eq. (8) and $\eta_{e}=g\left(\theta_{\mathrm{e}}\right)$, although the expression for $\Gamma$ is too lengthy to write here. However, the functional dependence of the "delay" of the Freedericksz transition on the shear rate $\zeta$ is clear to see from the form of $E_{c}$.

Although this Rapid Communication concerns a dual frequency nematic, allowing self-consistent determination of the relevant viscosity parameters, the same flow-delayed Freedericksz transition would also be predicted to occur in both standard nematic liquid crystals and in other liquid crystal systems. Our experimental technique has the potential to be extended for liquid crystals in other ways: through alternative surface treatments, leading to competition between alignment, flow, and electric fields; cholesteric liquid crystals where the internal chiral helix structure may compete with the flow and electric field; and lyotropic and aqueous chromonic systems, where positional ordering will affect the flow-electric-field competition.

In fact, our conclusions apply more generally to any system where orthogonal electric and flow forces compete to act to reorient an entity with anisotropic polarizability, for example, where carbon nanotubes are subjected to simultaneous electric fields and gas flows [24], or in electric-field-driven deposition of metallic or semiconductor nanorods from colloidal suspensions or evaporating solvents [25-27].

The research data associated with this paper is available at University of Strathclyde KnowledgeBase Ref. [28].

This work was supported by the Engineering and Physical Sciences Research Council (Grants No. EP/J009865/1 and No. EP/J009873/1).
[1] M. Miesowicz, Bull. Int. Acad. Pol. Sci. Lett. A 228 (1936).

[2] M. Miesowicz, Nature (London) 158, 27 (1946).

[3] E. Lueder, Liquid Crystal Displays: Addressing Schemes and Electro-Optical Effects (Wiley, New York, 2010).

[4] R. Zhao, B. Cumby, A. Russell, and J. Heikenfeld, Appl. Phys. Lett. 103, 223510 (2013).

[5] S. Xu, H. Ren, and S.-T. Wu, J. Phys. D: Appl. Phys. 46, 483001 (2013).

[6] C. W. Oseen, Trans. Faraday. Soc. 29, 883 (1933).

[7] F. C. Frank, Disc. Faraday. Soc. 25, 19 (1958).

[8] J. L. Ericksen, Arch. Rat. Mech. Anal. 4, 231 (1960).

[9] F. M. Leslie, Arch. Rat. Mech. Anal. 28, 265 (1968).

[10] P. G. de Gennes and J. Prost, The Physics of Liquid Crystals, 2nd ed. (Oxford University Press, New York, 1995).

[11] I.-K. Yang and A. D. Shine, J. Rheol. 36, 1079 (1992).

[12] K. Yoshida, M. Kikuchi, J.-H. Park, and S. Yokota, Sens. Actuators, A 95, 227 (2002).

[13] K. Negita, J. Chem. Phys. 105, 7837 (1996).

[14] M. De Volder, K. Yoshida, S. Yokota, and D. Reynaerts, J. Micromech. Microeng. 16, 612 (2006).

[15] J. A. Reyes, A. Corella-Madueo, and C. I. Mendoza, J. Chem. Phys. 129, 084710 (2008).

[16] P. Patrcio, C. R. Leal, L. F. V. Pinto, A. Boto, and M. T. Cidade, Liq. Cryst. 39, 25 (2012).
[17] J. Ananthaiah, R. Sahoo, M. V. Rasna, and S. Dhara, Phys. Rev. E 89, 022510 (2014).

[18] N. J. Mottram and C. V. Brown, Phys. Rev. E 74, 031703 (2006).

[19] J. L. Ericksen, Trans. Soc. Rheol. 5, 23 (1961).

[20] M. Kaiser and W. Pesch, Phys. Rev. E 48, 4510 (1993).

[21] I. W. Stewart, The Static and Dynamic Continuum Theory of Liquid Crystals (Taylor \& Francis, London, 2004).

[22] MATLAB and Statistics Toolbox Release 2015a, The MathWorks, Inc., Natick, MA.

[23] S. A. Jewell and J. R. Sambles, Phys. Rev. E 73, 011706 (2006)

[24] Y. Zhang, A. Chang, J. Cao, Q. Wang, W. Kim, Y. Li, N. Morris, E. Yenilmez, J. Kong, and H. Dai, Appl. Phys. Lett. 79, 3155 (2001).

[25] M. Mohammadimasoudi, L. Penninck, T. Aubert, R. Gomes, Z. Hens, F. Strubbe, and K. Neyts, Proc. SPIE 9170, 917013 (2014).

[26] K. M. Ryan, A. Mastroianni, K. A. Stancil, H. Liu, and A. P. Alivisatos, Nano Lett. 6, 1479 (2006).

[27] B. M. I. van der Zande, G. J. M. Koper, and H. N. W. Lekkerkerker, J. Phys. Chem. B 103, 5754 (1999).

[28] University of Strathclyde KnowledgeBase http://dx.doi.org/ 10.15129/335336c7-ce02-443c-b1c5-531eb273c7e6. 